\title{
A diagnostic support tool for lumbar spinal stenosis: a self-administered, self-reported history questionnaire Shin-ichi Konno*1, Shin-ichi Kikuchi ${ }^{1}$, Yasuhisa Tanaka ${ }^{2}$, Ken Yamazaki ${ }^{3}$, You-ichi Shimada ${ }^{4}$, Hiroshi Takei ${ }^{5}$, Toru Yokoyama ${ }^{6}$, Masahiro Okada ${ }^{6}$ and Shou-ichi Kokubun ${ }^{2}$
}

\begin{abstract}
Address: ${ }^{1}$ Department of Orthopaedic Surgery, Fukushima Medical University School of Medicine, Fukushima City, Fukushima, Japan, ${ }^{2}$ Department of Orthopaedic Surgery, Tohoku University, Sendai City, Miyagi, Japan, ${ }^{3}$ Department of Orthopaedic Surgery, Iwate Medical College, Morioka City, Iwate, Japan, ${ }^{4}$ Department of Orthopaedic Surgery, Akita University, Akita City, Akita, Japan, ${ }^{5}$ Department of Orthopaedic Surgery, Yamagata University, Yamagata City, Yamagata, Japan and 'Department of Orthopaedic Surgery, Hirosaki University, Hirosaki City, Aomori, Japan Email: Shin-ichi Konno* - skonno@fmu.ac.jp; Shin-ichi Kikuchi - sinichk@fmu.ac.jp; Yasuhisa Tanaka - ytanaka@tohoku-ctr-hsp.com; Ken Yamazaki - yamaken@iwate-med.ac.jp; You-ichi Shimada - yshimada@med.akita-u.ac.jp; Hiroshi Takei - htakei@med.id.yamagata-u.ac.jp; Toru Yokoyama - toru2000@cc.hirosaki-u.ac.jp; Masahiro Okada - akihiro@actv.ne.jp; Shou-ichi Kokubun - kokubun@nishitaga-hp.go.jp

* Corresponding author
\end{abstract}

Published: 30 October 2007

BMC Musculoskeletal Disorders 2007, 8:102 doi:10.1 186/147I-2474-8-102
Received: 16 April 2007

Accepted: 30 October 2007

This article is available from: http://www.biomedcentral.com/I47I-2474/8/102

(c) 2007 Konno et al; licensee BioMed Central Ltd.

This is an Open Access article distributed under the terms of the Creative Commons Attribution License (http://creativecommons.org/licenses/by/2.0), which permits unrestricted use, distribution, and reproduction in any medium, provided the original work is properly cited.

\begin{abstract}
Background: There is no validated gold-standard diagnostic support tool for LSS, and therefore an accurate diagnosis depends on clinical assessment. Assessment of the diagnostic value of the history of the patient requires an evaluation of the differences and overlap of symptoms of the radicular and cauda equina types; however, no tool is available for evaluation of the LSS category. We attempted to develop a self-administered, self-reported history questionnaire as a diagnostic support tool for LSS using a clinical epidemiological approach. The aim of the present study was to use this tool to assess the diagnostic value of the history of the patient for categorization of LSS.

Methods: The initial derivation study included 137 patients with LSS and 97 with lumbar disc herniation who successfully recovered following surgical treatment. The LSS patients were categorized into radicular and cauda equina types based on history, physical examinations, and MRI. Predictive factors for overlapping symptoms between the two types and for cauda equina symptoms in LSS were derived by univariate analysis. A self-administered, self-reported history questionnaire (SSHQ) was developed based on these findings. A prospective derivation study was then performed in a series of II 5 patients with LSS who completed the SSHQ before surgery. All these patients recovered following surgical treatment. The sensitivity of the SSHQ was calculated and clinical prediction rules for LSS were developed. A validation study was subsequently performed on 250 outpatients who complained of lower back pain with or without leg symptoms. The sensitivity and specificity of the SSHQ were calculated, and the test-retest reliability over two weeks was investigated in 217 patients whose symptoms remained unchanged.
\end{abstract}

Results: The key predictive factors for overlapping symptoms between the two categories of LSS were age $>50$, lower-extremity pain or numbness, increased pain when walking, increased pain when standing, and relief of symptoms on bending forward (odds ratio $\geq 2, p<0.05$ ). The key predictive factors for cauda equina type symptoms were numbness around the buttocks, walking almost causes urination, a burning sensation around the buttocks, numbness in the soles of both 
feet, numbness in both legs, and numbness without pain (odds ratio $\geq 2, p<0.05$ ). The sensitivity and specificity of the SSHQ were $84 \%$ and $78 \%$, respectively, in the validation data set. The area under the receiver operating characteristic curve was 0.797 in the derivation set and 0.782 in the validation data set. In the test-retest analysis, the intraclass correlation coefficient for the first and second tests was $85 \%$.

Conclusion: A new self-administered, self-reported history questionnaire was developed successfully as a diagnostic support tool for LSS.

\section{Background}

Lumbar spinal stenosis (LSS) is a well-recognized spinal disorder and a term used to describe a complex set of symptoms, physical findings, and radiological abnormalities caused by a narrowed spinal canal. The presence of a narrow canal in radiographic imaging does not in itself define the syndrome, and a diagnosis of LSS is defined by symptoms and clinical findings that must be supported by radiographic evidence. Computed tomography and magnetic resonance imaging are often non-specific and there may be discrepancies between clinical symptoms and imaging findings in cases of LSS [1-3].

There is no validated gold-standard diagnostic support tool for LSS, and therefore an accurate diagnosis depends on clinical assessment. However, there are few scientific evaluations of the sensitivity and specificity of diagnoses based on clinical history and physical examinations, or appropriate correlations of these data with imaging and operative findings. Katz et al. used the opinion of two expert orthopedic surgeons to define the presence or absence of LSS [4], and found that the factors in the patient history that were most strongly associated with diagnosis of LSS were a higher age, severe lower-extremity pain, and the absence of pain when seated. The physical findings most strongly associated with the diagnosis were a wide-based gait, an abnormal Romberg test, thigh pain following 30 seconds of lumbar extension, and neuromuscular deficits.

There are two categories of leg symptoms caused by LSS [5]. One type of stenosis presents as unilateral radicular pain (the radicular type), with symptoms of pain, burning, numbness and paresthesia following a specific dermatome or dermatomes. The fifth lumbar nerve root associated with L5 stenosis is most commonly involved. The other type of LSS has symptoms with less dermatomal-specific neurogenic claudication, and nerve roots below L5 are most commonly involved. The typical patient presents with complaints of aching, cramping, or a burning sensation in the bilateral legs. Occasionally, numbness is also apparent and some patients complain of bladder dysfunction and sexual difficulties.
Full-blown cauda equina syndrome only occurs in rare instances, but the above symptoms can occur as a part of cauda equina syndrome [6,7]. Therefore, we hypothesized that leg symptoms in LSS might be divided into two categories: a radicular type and a cauda equina type. There are significant differences between the symptoms of these types, but there is also significant overlap between the symptoms. Since both the central canal and foraminal dimensions increase in flexion and diminish in extension, patients with both types of LSS experience exacerbation of symptoms with extension and improvement with flexion.

Assessment of the diagnostic value of the history of the patient requires an evaluation of the differences and overlap of symptoms of the radicular and cauda equina types; however, no tool is available for evaluation of the LSS category. Therefore, we attempted to develop a self-administered, self-reported history questionnaire as a diagnostic support tool for LSS using a clinical epidemiological approach. The aim of the present study was to use this tool to assess the diagnostic value of the history of the patient for categorization of LSS.

\section{Methods}

\section{Derivation study I}

A series of 137 patients with LSS and 97 with lumbar disc herniation who successfully recovered following surgical treatment in our department during 2000 and 2003 were included in this study (Table 1). Patients with cervical

Table I: Demographic data for patients in derivation study I

\begin{tabular}{lcc}
\hline & $\begin{array}{c}\text { LSS } \\
(\mathrm{n}=137)\end{array}$ & $\begin{array}{c}\text { LDH } \\
(\mathrm{n}=97)\end{array}$ \\
\hline Male (\%) & 46 & 58 \\
Female (\%) & 54 & 42 \\
Mean age (yr) & 68 & 41 \\
Mean duration of symptoms (mo) & 21 & 5 \\
Cauda equina type intermittent claudication & 50 & - \\
Radicular type intermittent claudication & 87 & - \\
Findings on MRI & & 14 \\
$\quad$ One level stenosis & 102 & 0 \\
Two level stenosis & 23 & 0 \\
Three level stenosis & 12 &
\end{tabular}


myelopathy, diabetic neuropathy, previous surgery, peripheral vascular disease, inflammatory disorders, and degenerative scoliosis (defined as lateral tilting of more than 10 degrees) were excluded. Each patient was evaluated by the study investigators using a standard protocol. Operative and follow-up visit notes were reviewed to determine if stenosis was confirmed intraoperatively and if symptoms improved following surgery. Nerve root compression resulting exclusively from a herniated nucleus pulposus was not considered as a symptom of LSS.

Assessment of history included questions on location, frequency and severity of pain, and on symptoms including numbness, tingling, and provocative factors. The physical examination included a gait-loading test to confirm neurogenic intermittent claudication; this test involves assessment of walking capacity and symptoms, and a neurological examination of motor, sensory, and reflex activity [8]. We investigated symptoms during gait loading and neurological findings just after gait loading. Reflexes were graded from 0 (no response) to 4 (clonus) at the Achilles tendon and patellar tendon, and strength was graded from 0 (no movement) to 5 (normal) at the knee flexors and extensors, ankle dorsiflexors and plantar flexors, and extensor hallucis longus. A pinprick sensation was graded as absent, decreased, or normal at the dorsomedial foot, dorsolateral foot, medial calf, and lateral calf. MRI radiographical reports were abstracted from patient records.

The LSS patients were categorized into radicular and cauda equina types based on history, physical examination, and MRI findings. The radicular type was characterized by symptoms of pain, burning, numbness, and paresthesias following a specific dermatome with radiological evidence of the responsible nerve root compression, which was confirmed if intermittent claudication was abolished following single nerve root infiltration. Patients of the cauda equina type presented some bilateral symptoms related to cauda equina compression syndrome with less dermatomal-specific neurogenic claudication and radiological evidence of cauda equina compression. The cauda euqina type spinal stenosis is distinct from the cauda equina syndrome. A full blown cauda equina syndrome occurs in rare instances in the cauda equina type spinal stenosis. Therefore, urgent surgery is not required in the cauda equina type spinal stenosis. Predictive factors for overlap symptoms between the two types of LSS were derived from the data and factors for predicting the cauda equina type were also determined. Based on the results of univariate analysis for predictors of LSS, a self-administered, self-reported history questionnaire (SSHQ) was developed as a diagnostic support tool for LSS.

\section{Derivation study 2}

This study was performed in six university hospitals, ten medical centers, and thirty one hospitals and clinics affiliated with university hospitals or medical centers during January and March in 2004. A series of 115 patients with LSS gave informed consent to participate in the study and answered the SSHQ before surgery. All these patients recovered following surgery. Patients with cervical myelopathy, diabetic neuropathy, previous surgery, inflammatory disorders, and degenerative scoliosis were excluded. All patients were evaluated by study investigators using the same protocol as that in derivation study 1 . Operative and follow-up visit notes were reviewed to determine if stenosis was confirmed intraoperatively and if symptoms improved following surgery. Nerve root compression resulting exclusively from a herniated nucleus pulposus was not considered as a part of LSS syndrome. All LSS patients were categorized into radicular or cauda equina types based on history, physical examination, and MRI findings using the same criteria as those in derivation study 1 . There were 55 patients with radicular type LSS and 60 patients with the cauda equina type (Table 2). A responsible nerve root was confirmed if intermittent claudication was abolished following single nerve root infiltration. The sensitivity of each question on the SSHQ was calculated and compared between the radicular and cauda equina types. To assess the cut-off point to distinguish between the types, one point was assigned to each question on the SSHQ, and the clinical prediction rule was defined based on the scores.

\section{Validation study}

We prospectively evaluated the association between the diagnosis of LSS and clinical information, including the history and physical examination of patients with leg symptoms. This study was performed in six university hospitals, ten medical centers, and sixty eight hospitals and clinics affiliated with university hospitals or medical centers during July and September in 2004. We enrolled consecutive patients older than 20 years of age with primary symptoms of pain or numbness in the legs. We excluded patients who have been treated by some medical

Table 2: Demographic data for patients in derivation study 2

\begin{tabular}{lcc}
\hline & $\begin{array}{c}\text { Radicular type } \\
(\mathrm{n}=55)\end{array}$ & $\begin{array}{c}\text { Cauda equina type } \\
(\mathrm{n}=60)\end{array}$ \\
\hline Male (\%) & 52 & 42 \\
Female (\%) & 48 & 58 \\
Mean age (yr) & 68 & 71 \\
Mean duration of symptoms (mo) & 19 & 32 \\
Findings on MRI & & \\
$\quad$ One level stenosis & 43 & 47 \\
Two level stenosis & 12 & 10 \\
Three level stenosis & 0 & 3
\end{tabular}


practices within one year before examination. Patients with cervical myelopathy, previous surgery, degenerative scoliosis (defined as lateral tilting of more than 10 degrees) and inflammatory disorders were also excluded. This study included 250 patients who complained of leg symptoms, including cases of LSS $(\mathrm{n}=165)$, lumbar disc herniation $(n=61)$, diabetic neuropathy $(n=13)$, and peripheral vascular disease $(\mathrm{n}=11)$ (Table 3$)$. The study was approved by the institutional review board of each study institution as necessary. Written informed consent was obtained from the all patients. The patients gave informed consent and then answered the SSHQ. The following steps were taken to reach a final diagnosis for each of the enrolled patients (Figure 1). In the first step, at each institution the orthopedic physician who saw a patient made the clinical diagnosis based on the history, physical examination, and radiographic findings. In addition, to verify the diagnosis made by each physician, six boardcertified spine surgeons approved by the Japanese Board of Spine Surgery also made a diagnosis for each patient based on the clinical information and findings of the MRI. The opinions of six board-certified spine surgeons approved by the Japanese Board of Spine Surgery were used as the gold standard for diagnosis of LSS. The radicular type was characterized by symptoms of pain, burning, numbness, and paresthesias following a specific dermatome with radiological evidence of the responsible nerve root compression, which was confirmed if intermittent claudication was abolished following single nerve root infiltration. Patients of the cauda equina type presented some bilateral symptoms related to cauda equina compression syndrome with less dermatomal-specific neurogenic claudication and radiological evidence of cauda equina compression.

The sensitivity, specificity, likelihood ratio, and area under the receiver operating characteristic (ROC) curve

Table 3: Demographic data for patients in the validation study

\begin{tabular}{lcc}
\hline & LSS $(\mathrm{n}=165)$ & $\begin{array}{c}\text { The others } \\
(\mathrm{n}=85)\end{array}$ \\
\hline Male (\%) & 47 & 51 \\
Female (\%) & 53 & 49 \\
Mean age (yr) & 71 & 48 \\
Mean duration of symptoms (mo) & 28 & 24 \\
Clinical impressions of patient & $\begin{array}{c}\text { Cauda equina } \\
\text { type 78 }\end{array}$ & $\mathrm{LDH}^{*} 61$ \\
condition & Radicular type 87 & $\mathrm{DN}^{*} 13$ \\
& & $\mathrm{PAD}^{*} 1 \mathrm{I}$ \\
Findings on MRI & & 12 \\
$\quad$ One level stenosis & 127 & 2 \\
$\quad$ Two level stenosis & 31 & 0 \\
Three level stenosis & 7 & \\
\hline
\end{tabular}

* LDH: Lumbar Disc Herniation, DN: Diabetic Neuropathy, PAD:

Peripheral Artery Disease

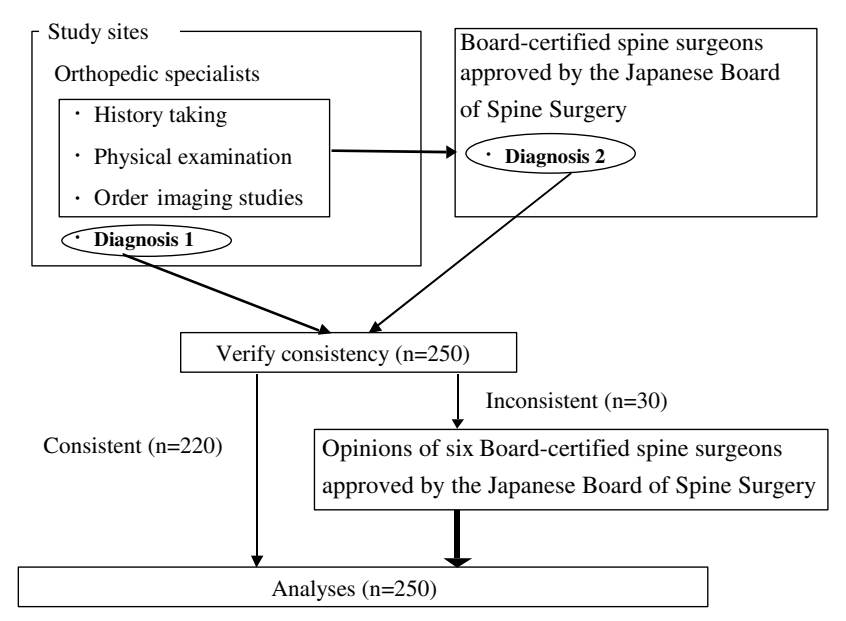

Figure I

Flow chart of how the diagnosis of LSS was determined.

were estimated. 217 patients classified by investigators as suffering from lower back pain without a significant change in symptoms were given the SSHQ two weeks later during an outpatient visit, and the test-retest reliability over two weeks was investigated in these patients.

\section{Statistical analysis}

History and physical examination variables were dichotomized at clinically sensible cut-off values. Pinprick, strength, and Achilles reflexes were each classified as always normal or with at least 1 abnormal finding. Univariate analyses were performed to derive predictors of LSS using logistic regression analysis. Two-by-two contingency tables were prepared to calculate the sensitivity, specificity, and likelihood ratio of the SSHQ. The area under the ROC curve for the derivation data set was estimated to investigate the internal validity of the clinical prediction rule, and the area under the ROC curve for the validation data set was estimated to examine the external validity. Reliability was investigated based on the reproducibility in the test-retest method. Test-retest analysis was performed in 217 patients with a 14-day period between the first and second tests. Test-retest data were examined graphically by plotting the difference between tests against the mean of the 2 tests [9]. The intraclass correlation coefficient of the SSHQ score for the first and second tests was calculated to confirm reproducibility. The $\kappa$ coefficient was calculated to examine conformity for each item, based on the following criteria: 0 to $<0.2$, poor; 0.2 to $<0.4$, fair; 0.4 to $<0.6$, moderate; 0.6 to $<0.8$, substantial; and $>0.8$, almost perfect [10]. All the studies were approved by the ethics committee of Fukushima Medical University. 


\section{Results \\ Univariate analysis for predictors of LSS}

Key factors for predicting overlapping symptoms between the two types of LSS are shown in Table 4. Five history findings had an odds ratio $\geq 2$ or $\mathrm{p}<0.05$ : age $>50$, lowerextremity pain or numbness, increased pain when walking, increased pain when standing, and improvement of symptoms on bending forward. No physical examination finding had an odds ratio $\geq 2$ or $\mathrm{p}<0.05$. There was no significant difference in the odds ratio of all the predictive factors except for age. Key factors for predicting the cauda equina type of LSS are shown in Table 5. Six history findings had an odds ratio $\geq 2$ or $\mathrm{p}<0.05$ : numbness around the buttocks, walking nearly causes urination, a burning sensation around the buttocks, numbness in the soles of both feet, numbness in both legs, and numbness without pain. Physical examination findings with an odds ratio $\geq$
2 or $\mathrm{p}<0.05$ included the absence of or a weak Achilles reflex response.

\section{A self-administered, self-reported history questionnaire (SSHQ)}

Based on the results of univariate analysis for predictors of LSS, we developed the SSHQ as a diagnostic support tool for LSS (see Additional File 1 and 2). The SSHQ included the following questions:

Q1: Numbness and/or pain in the thighs down to the calves and shins.

Q2: Numbness and/or pain increase in intensity after walking for a while, but are relieved by taking a rest.

Q3: Standing for a while brings on numbness and/or pain in the thighs down to the calves and shins.

Table 4: Univariate analyses for factors from the MD and MRI data sheets associated with a diagnosis of LSS

\begin{tabular}{|c|c|c|c|c|c|}
\hline & LSS (-) (n = 97) & LSS $(+)(n=137)$ & Odds Ratio & $95 \% \mathrm{Cl}$ & $\mathrm{P}$-value \\
\hline Age (years) $>50$ & $20.6 \%$ & $94.9 \%$ & 71.50 & $28.9-176.9$ & $<0.001$ \\
\hline Gender (Female) & $42.0 \%$ & $54.0 \%$ & 1.60 & $0.95-2.71$ & 07 \\
\hline \multicolumn{6}{|l|}{ Symptoms } \\
\hline Leg pain or numbness $(+)$ & $87.6 \%$ & $94.9 \%$ & 2.62 & $0.99-6.93$ & 0.045 \\
\hline Low back pain $(+)$ & $72.2 \%$ & $65.0 \%$ & 0.72 & $0.41-1.26$ & 0.245 \\
\hline Worse when walking but relieved by taking a rest & $18.6 \%$ & $94.2 \%$ & 70.77 & $29.39-170.4$ & $<0.001$ \\
\hline Numbness in both legs $(+)$ & $15.5 \%$ & $24.8 \%$ & 1.80 & $0.92-3.54$ & 0.083 \\
\hline Numbness in the soles of both feet $(+)$ & $13.4 \%$ & $20.4 \%$ & 1.66 & $0.81-3.40$ & 0.163 \\
\hline Numbness around the buttocks $(+)$ & $9.3 \%$ & $15.3 \%$ & 77 & $77-4.05$ & 173 \\
\hline Numbness without pain & $8.2 \%$ & $11.7 \%$ & 1.53 & $0.63-3.75$ & 0.344 \\
\hline A burning sensation around the buttocks & $6.2 \%$ & $8.2 \%$ & 0.94 & $0.32-2.80$ & 0.912 \\
\hline Walking nearly causes urination & $3.1 \%$ & $5.1 \%$ & 1.69 & $0.43-6.70$ & 0.452 \\
\hline Worse when standing for a while & $24.7 \%$ & $84.7 \%$ & 11.38 & $6.20-20.91$ & $<0.001$ \\
\hline Symptoms improve on bending forward & $8.1 \%$ & $72.3 \%$ & 25.47 & $11.66-55.64$ & $<0.001$ \\
\hline \multicolumn{6}{|l|}{ Physical Examination } \\
\hline Straight Leg Raising test positive & $33.0 \%$ & $21.9 \%$ & 0.57 & $0.32-1.02$ & 0.058 \\
\hline $\begin{array}{l}\text { Symptoms induced by having patients bend } \\
\text { forward }(+)\end{array}$ & $30.9 \%$ & $20.4 \%$ & 0.57 & $0.32-1.04$ & 0.067 \\
\hline $\begin{array}{l}\text { Symptoms induced by having patients bend } \\
\text { backward (+) }\end{array}$ & $53.6 \%$ & $62.0 \%$ & 1.38 & $0.81-2.35$ & 0.229 \\
\hline Abnormal manual muscle strength test I) & $8.2 \%$ & $10.2 \%$ & 1.27 & $0.51-3.15$ & 0.611 \\
\hline \multicolumn{6}{|l|}{ Sensory disturbance } \\
\hline$(-)$ & $57.7 \%$ & $49.6 \%$ & reference & & \\
\hline$(+) 2)$ & $37.1 \%$ & $45.3 \%$ & 1.40 & $0.82-2.38$ & 0.214 \\
\hline Missing data & $5.2 \%$ & $5.1 \%$ & 0.99 & $0.30-3.22$ & 0.988 \\
\hline \multicolumn{6}{|l|}{ Achilles tendon reflex } \\
\hline Normal & $51.5 \%$ & $48.2 \%$ & reference & & \\
\hline Abnormal 3) & $43.3 \%$ & $46.7 \%$ & 1.15 & $0.68-1.94$ & 0.605 \\
\hline Missing data & $5.2 \%$ & $5.1 \%$ & 0.99 & $0.30-3.22$ & 0.988 \\
\hline \multicolumn{6}{|l|}{ Patellar tendon reflex } \\
\hline Normal & $70.1 \%$ & $62.8 \%$ & reference & & \\
\hline Abnormal 3) & $24.7 \%$ & $32.1 \%$ & 1.44 & $0.80-2.58$ & 0.221 \\
\hline Missing data & $5.2 \%$ & $5.1 \%$ & 0.99 & $0.30-3.22$ & 0.988 \\
\hline
\end{tabular}

I) $M M T<=3$, Strength was graded from 0 (no movement) to 5 (normal) at the knee extensors, ankle dorsiflexors, and plantar flexors, and extensor hallucis longus.

2) Hypoesthesia, analgesia, or hyperalgesia at the medial knee, dorsal foot, plantar foot, and perineal lesion

3) Absence or low response of deep reflexes 
Table 5: Univariate analyses for factors from the MD and MRI data sheets associated with a diagnosis of the cauda equina type of LSS

\begin{tabular}{|c|c|c|c|c|c|}
\hline & $\begin{array}{l}\text { Radicular type } \\
\quad(n=87)\end{array}$ & $\begin{array}{l}\text { Cauda Equina type } \\
\qquad(\mathrm{n}=50)\end{array}$ & Odds Ratio & $95 \% \mathrm{Cl}$ & p-value \\
\hline Age $($ years) $>50$ & $94.3 \%$ & $96.0 \%$ & 1.46 & $0.27-7.84$ & 0.655 \\
\hline Gender (Female) & $51.7 \%$ & $56.0 \%$ & 1.20 & $0.60-2.38$ & 0.608 \\
\hline \multicolumn{6}{|l|}{ Symptoms } \\
\hline Leg pain or numbness $(+)$ & $97.7 \%$ & $96.0 \%$ & 0.56 & $0.08-4.14$ & 0.569 \\
\hline Low back pain $(+)$ & $65.5 \%$ & $62.0 \%$ & 0.86 & $0.42-1.77$ & 0.679 \\
\hline Worse when walking but relieved by taking a rest & $96.6 \%$ & $96.0 \%$ & 0.86 & $0.14-5.31$ & 0.868 \\
\hline Numbness in both legs $(+)$ & $11.5 \%$ & $82.0 \%$ & 35.08 & $13.20-93.19$ & $<0.001$ \\
\hline Numbness in the soles of both feet $(+)$ & $6.9 \%$ & $78.0 \%$ & 47.86 & $16.49-138.9$ & $<0.001$ \\
\hline Numbness around the buttocks $(+)$ & $1 \mathrm{l} .5 \%$ & $68.0 \%$ & 6.36 & $6.74-39.73$ & $<0.001$ \\
\hline Numbness without pain & $10.3 \%$ & $37.0 \%$ & 5.31 & $2.17-13.0 \mid$ & $<0.001$ \\
\hline A burning sensation around the buttocks & $6.9 \%$ & $34.0 \%$ & 6.95 & $2.52-19.19$ & $<0.001$ \\
\hline Walking nearly causes urination & $4.6 \%$ & $26.0 \%$ & 7.29 & $2.23-23.86$ & $<0.001$ \\
\hline Worse when standing for a while & $92.0 \%$ & $82.0 \%$ & 0.40 & $0.14-1.15$ & 0.08 \\
\hline Symptoms improve on bending forward & $86.2 \%$ & $74.0 \%$ & 0.46 & $0.19-1.10$ & 0.07 \\
\hline \multicolumn{6}{|l|}{ Physical Examination } \\
\hline Straight Leg Raising test positive & $21.8 \%$ & $22.0 \%$ & 1.01 & $0.44-2.34$ & 0.983 \\
\hline Symptoms induced by having patients bend forward $(+)$ & $19.5 \%$ & $22.0 \%$ & 1.16 & $0.49-2.73$ & 0.731 \\
\hline Symptoms induced by having patients bend backward $(+)$ & $63.2 \%$ & $58.0 \%$ & 0.80 & $0.39-1.64$ & 0.546 \\
\hline Abnormal manual muscle strength test I) & $9.2 \%$ & $12.0 \%$ & 1.35 & $0.44-4.13$ & 0.602 \\
\hline \multicolumn{6}{|l|}{ Sensory disturbance } \\
\hline$(-)$ & $49.4 \%$ & $50.0 \%$ & reference & & \\
\hline$(+) 2)$ & $43.7 \%$ & $46.0 \%$ & 1.40 & $0.82-2.38$ & 0.214 \\
\hline Missing data & $6.9 \%$ & $4.0 \%$ & 0.56 & $0.11-2.90$ & 0.486 \\
\hline \multicolumn{6}{|l|}{ Achilles tendon reflex } \\
\hline Normal & $65.5 \%$ & $30.0 \%$ & reference & & \\
\hline Abnormal 3) & $27.6 \%$ & $66.0 \%$ & 5.10 & $2.41-10.79$ & $<0.001$ \\
\hline Missing data & $6.9 \%$ & $4.0 \%$ & 0.56 & $0.11-2.90$ & 0.486 \\
\hline \multicolumn{6}{|l|}{ Patellar tendon reflex } \\
\hline Normal & $63.2 \%$ & $62.0 \%$ & reference & & \\
\hline Abnormal 3) & $29.9 \%$ & $34.0 \%$ & 1.21 & $0.57-2.54$ & 0.617 \\
\hline Missing data & $6.9 \%$ & $4.0 \%$ & 0.56 & $0.11-2.90$ & 0.486 \\
\hline
\end{tabular}

I) $M M T<=3$, Strength was graded from 0 (no movement) to 5 (normal) at the knee extensors, ankle dorsiflexors, and plantar flexors, and extensor hallucis longus.

2) Hypoesthesia, analgesia, or hyperalgesia at the medial knee, dorsal foot, plantar foot, and perineal lesion

3) Absence or low response of deep reflexes

Q4: Numbness and/or pain are reduced by bending forward.

The key questions for diagnosis of cauda equina symptoms were as follows:

Q5: Numbness is present in both legs.

Q6: Numbness is present in the soles of both feet.

Q7: Numbness arises around the buttocks.

Q8: Numbness is present, but pain is absent.

Q9: A burning sensation arises around the buttocks.

\section{Clinical prediction rule}

The sensitivity of each question in the derivation study was calculated for the radicular and cauda equina types of LSS. The sensitivity differed significantly between the categories (Figure 2). To assess the cut-off point to distinguish between the two types, each question was assigned one point. The scores of predictors of cauda equina symptoms (Q5-Q10) were significantly different between the categories, and the cut-off point was two (Figure 3). Based on these results, a clinical prediction rule was defined based on the total scores: a score of 4 points on Q1-Q4 indicates the presence of LSS; a score of 4 on Q1-Q4 and $<1$ on Q5-Q10 indicates the radicular type of LSS; and a score of $>1$ on Q1-Q4 and > 2 on Q5-Q10 indicates the cauda equina type of LSS.

Q10: Walking nearly causes urination. 


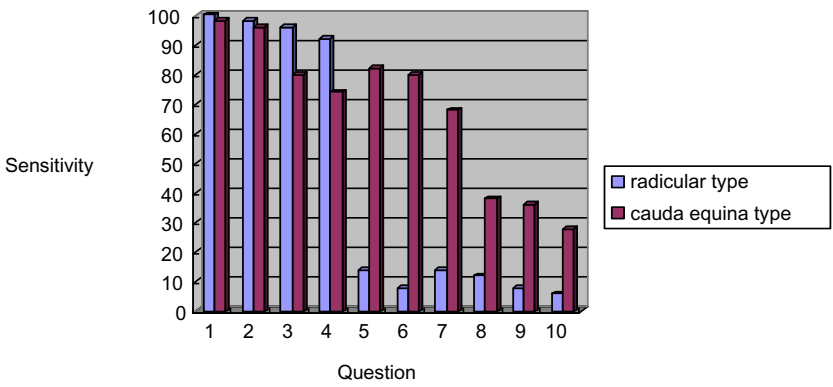

Figure 2

Comparison of the sensitivity of each question for radicular and cauda equina types of LSS.

\section{Validity}

It took respondents an average of about 1 minute to answer the 10 questions on the SSHQ. Performance indices for the clinical prediction rule are shown in Table 6 . The area under the ROC curve was 0.797 in the derivation set and 0.782 in the validation data set (Figure 4). These findings indicate that the SSHQ has both internal and external validity as a diagnostic tool for LSS. The difference between tests plotted against the mean of the tests indicated no obvious relationship or bias (Figure 5). The intraclass correlation coefficient of the SSHQ score for the first and second tests was 0.85 , which indicates sufficient reproducibility. One item of the $\kappa$ coefficient was found to be "fair" (question 8), and all other items were rated as having a conformity of moderate or above.

\section{Discussion}

Spinal stenosis patients frequently present with few objective physical findings. Jonsson and Stromqvist found that about $65 \%$ of patients have decreased walking ability [11], but up to $95 \%$ of patients treated surgically have only subjective symptoms, principally pain $[12,13]$. Furthermore, diagnostic imaging cannot be used reliably to diagnose LSS, since CT and MRI are often non-specific and

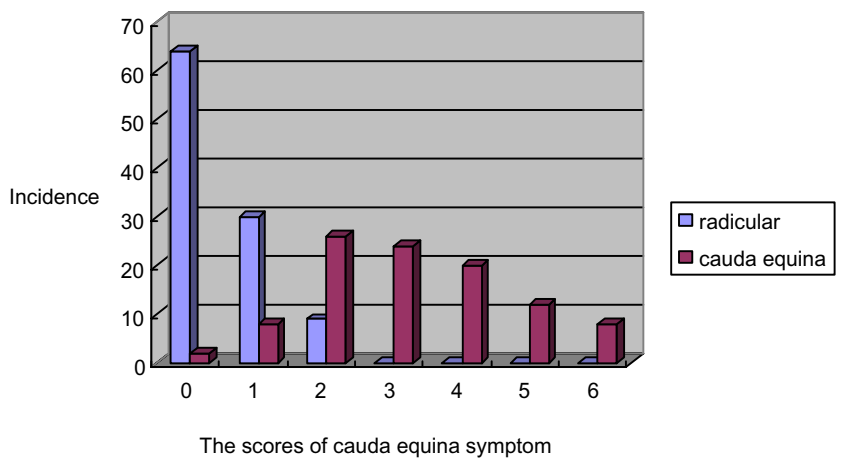

Figure 3

Cut-off point to distinguish between the categories.

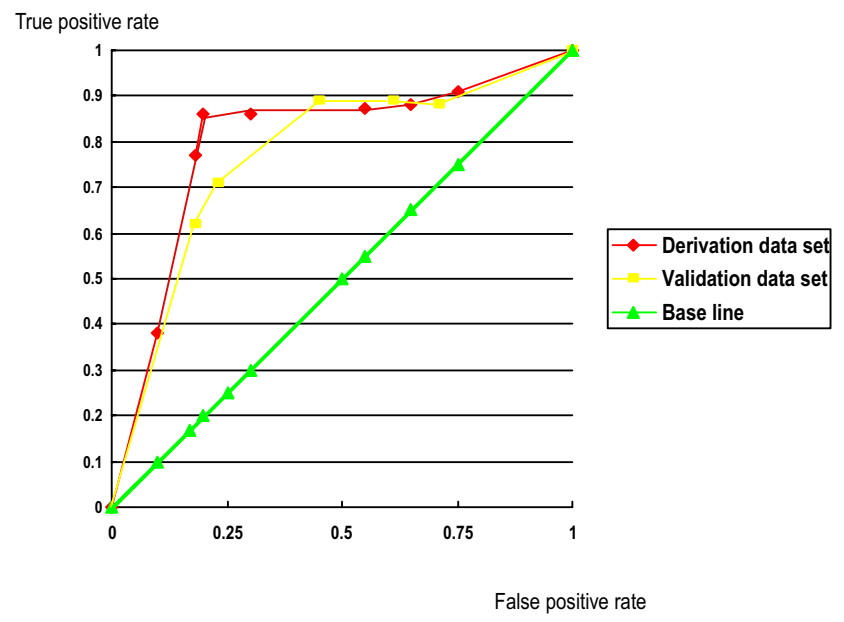

Derivation Data Set : ROC Area=0.797 (95\%Cl, 0.714-0.862) Validation Data Set : ROC Area $=0.782(95 \% \mathrm{Cl}, 0.685-0.869)$

\section{Figure 4}

Receiver operating characteristic (ROC) curves for the derivation and validation datasets.

do not prove that symptoms arise from nerve root compression $[14,15]$. A systematic review of original diagnostic studies on LSS revealed that no firm conclusions about the diagnostic performance of the different tests could be drawn due to heterogeneity and overall poor quality[16].

We developed a simple clinical diagnostic support tool to identify patients with LSS[17]. Although further studies

Difference between test and retest

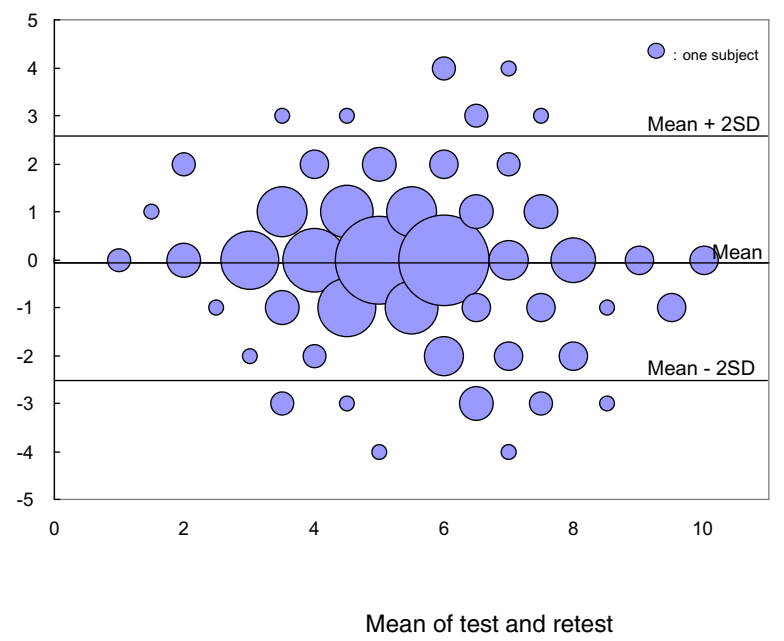

Figure 5

Scatter plot of differences versus the means of the test and the retest. 
Table 6: Performance indices for the clinical prediction rule

\begin{tabular}{lcc}
\hline \multirow{2}{*}{ Index } & \multicolumn{2}{c}{ Estimate } \\
& $\begin{array}{c}\text { Derivation Data } \\
\text { Set }(\mathrm{n}=234)\end{array}$ & $\begin{array}{c}\text { Validation Data } \\
\text { Set }(\mathrm{n}=250)\end{array}$ \\
\hline Sensitivity & 0.855 & 0.843 \\
Specificity & 0.791 & 0.781 \\
Likelihood Ratio & & \\
Positive Test Result & 1.951 & 1.886 \\
Negative Test Result & 0.201 & 0.214 \\
Area under the ROC curve & 0.797 & 0.782 \\
\hline
\end{tabular}

Positive criteria: Total score $\geq 4$ (QI-Q4) or Total score $\geq$ I (QIQ4) and $\geq 2$ (Q5-QI0)

are needed to validate this tool in primary care settings, it has a sensitivity of $92.8 \%$ and a specificity of $72.0 \%$. By asking patients, who presented with back and leg symptoms suggestive of LSS, to fill out a simple questionnaire, consisting of five questions on their medical history (age and history of diabetes) and symptoms (presence or absence of intermittent claudication, aggravation of symptoms by standing and relief of symptoms by forward bending) followed by a short clinical examination checking the postural changes in their leg symptoms, Achilles' tendon reflex, SLR test and the measurement of ABI, the diagnosis of LSS can be established by high sensitivity and spcificity without obtaining MRI. Therefore, misdiagnosis or underdianosis of LSS at the primary care levels can be minimized and patients have a greater chance to get access to appropriate medical service by a referral to a spine specialist. A self-administered, self-reported history questionnaire as a diagnostic support tool for LSS might be more useful for clinician or patients. Therefore, we attempted to develop a self-administered, self-reported history questionnaire as a diagnostic support tool for LSS using a clinical epidemiological approach. To make an accurate diagnosis of LSS from history findings, we categorized the condition into radicular and cauda equina types. A comparison of the sensitivity of each question on the SSHQ showed both overlap and differences between the two categories (Figure 1). These findings suggest that the category of LSS requires consideration to make an accurate diagnosis. The scoring system includes a cut-off point to distinguish the radicular type from the cauda equina type (Figure 2). About $50 \%$ of radicular-type cases show relief of symptoms at six months after nerve root block and a further $17 \%$ improve with more time after nerve root block. In the cauda equina type, nerve root block is not efficient in relieving the symptoms and therefore surgical intervention is recommended [18]. Therefore, we consider that it is important to define the type of neurogenic intermittent claudication before selecting the therapeutic method, since surgery may be avoidable in certain cases.
The 10 items on the SSHQ for diagnosis of LSS require answers of either "yes" or "no" to minimize any difficulty with responses. As noted above, it took respondents an average of about 1 minute to answer the questions, which indicates that the questionnaire was easy to understand. However, the study has several limitations. First, there is no gold standard for diagnosis of LSS, but in the absence of valid objective criteria we believe that expert opinion is a reasonable strategy for making a diagnosis of a clinical syndrome, and this approach has been used for a variety of disorders. Therefore, we used LSS diagnoses made by six board-certified spine surgeons in our validation study. Second, we did not use logistic regression and multivariate models. Based on the results of this paper, we are planning to use logistic regression and multivariate models in the next project according to STARD checklist for reporting diagnostic accuracy studies [19]. We also note that a larger prospective derivation and validation studies might reveal additional independent factors that correlate with diagnosis of LSS.

\section{Conclusion}

The newly developed self-administered, self-reported history questionnaire can be used for diagnosis of LSS with high sensitivity, specificity, and reproducibility.

\section{Competing interests}

The author(s) declare that they have no competing interests.

\section{Authors' contributions}

SKonno, SKikuchi, SKokuban, YT, YS, KY, MO, TY and HT conceived the study and participated in the study design. SK performed the statistical analysis. SK drafted the initial manuscript for journal submission and participated in revisions. SKonno, SKikuchi, SKokuban, YT, YS, KY, MO, TY and HT coordinated data collection at each site. All authors read and approved the final manuscript.

\section{Additional material}

\section{Additional file 1}

Japanese version of the SSHQ. A copy of the Japanese version of the SSHQ for Japanese readers

Click here for file

[http://www.biomedcentral.com/content/supplementary/1471-

2474-8-102-S1.doc]

\section{Additional file 2}

English version of the SSHQ. A copy of the English version of the SSHQ Click here for file

[http://www.biomedcentral.com/content/supplementary/14712474-8-102-S2.doc] 


\section{Acknowledgements}

This study was supported by Grants from the Fukushima Medical Research Promotion Foundation.

\section{References}

I. de Graaf I, Prak A, Bierma-Zeinstra S, Thomas S, Peul W, Koes B: Diagnosis of lumbar spinal stenosis: a systematic review of the accuracy of diagnostic tests. Spine 2006, 3 I: I | 68- I I 76.

2. Beattie PF, Meyers SP, Stratford P, Millard RW, Hollenberg GM: Associations between patient report of symptoms and anatomic impairment visible on lumbar magnetic resonance imaging. Spine 2000, 25:819-828.

3. Amundsen T, Weber H, Lilleas F, Nordal HJ, Abdelnoor M, Magnaes $\mathrm{B}$ : Lumbar spinal stenosis. Clinical and radiologic features. Spine 1995, 20:1178-1186.

4. Katz JN, Dalgas M, Stucki G, Katz NP, Bayley J, Fossel AH, Chang LC, Lipson S]: Degenerative lumbar spinal stenosis Diagnostic value of the history and physical examination. Arthritis \& Rheumatism 1995, 38: I236-I24I.

5. Mirkovic S, Lybulski G, Montgomery DM, Wang AM, Wesolowski DP, Garfin SR: Spinal stenosis Clinical Evaluation and Different Diagnosis. Rothman-Simeone, the spine 4th edition. 1999:796-806.

6. Rauschning W: Pathoanatomy of lumbar disc degeneration and stenosis. Acta Orthop Scand 1993, 64:3-12.

7. Yoshida M, Shima K, Taniguchi Y, Tamaki T, Tanaka T: Hypertrophied ligamentum flavum in lumbar spinal canal stenosis: Pathogenesis and morphologic and immunohistochemical observation. Spine 1992, I7:1353-1360.

8. Konno S, Kikuchi S: Prospective study of surgical treatment of degenerative spondylolisthesis. Comparison between decompression alone and decompression with Graf system stabilization. Spine 2000, 25:| 533-1537.

9. Bland JM, Altman DG: Statistical methods for assessing agreement between two methods of clinical measurement. Lancet 1986, I:307-10.

10. Chalmers I: Underreporting research is scientific misconduct. JAMA 1990, 263:|405-1408.

II. Jonnson B, Stromqvist B: Symptoms and signs in degeneration of the lumbar spine : a prospective, consecutive study of 300 operated patients. J Bone Joint Surg $\mathrm{Br}$ 1993, 75:381-384.

12. Pheasant HC, Dyck P: Failed lumbar disc surgery: cause, assessment, treatment. Clin Orthop Relat Res 1982, ( I 64):93-109.

13. Ray CD: Extensive lumbar decompression: Patient selection and results. In Lumbar spine surgery Edited by: White $\mathrm{AH}$, Rothman RH, Ray CD. St. Louis CV Mosby Co; 1987.

14. Boden SD, Davis DO, Dina TS, Partronas NJ, Wiesel SW: Abnormal magnetic resonance scans of the lumbar spine in asymptomatic subjects: a prospective investigation. J Bone Joint Surg [Am] 1990, 72:403-408.

15. Wiesel SW, Tsourmas N, Feffer HL, Citrin CM, Patronas N: A study of computer-assisted tomography. The incidence of positive CAT scans in an asymptomatic group of patients. Spine 1984, 9:549-55I.

16. de Graaf I, Prak A, Bierma-Zeinstra S, Thomas S, Peul W, Koes B: Diagnosis of lumbar spinal stenosis: a systematic review of the accuracy of diagnostic tests. Spine 2006, 3 I : I I68- I I 76

17. Konno S, Hayashino Y, Fukuhara S, Kikuchi S, Kaneda K, Seichi A, Chiba K, Satomi K, Nagata K, Kawai S: Development of a clinical diagnosis support tool to identify patients with lumbar spinal stenosis. Eur Spine J 2007 in press. [Epub ahead of print]

18. Konno S, Kikuchi S: Pathomechanism of spinal canal stenosis. The therapeutic effects of nerve root infiltration, selective spinal angiography, and sympathetic nerve block. Proceeding of the 30th congress of the international college of surgeons. Bologna 1996:1309-1313.

19. Bossuyt PM, Reitsma JB, Bruns DE, Gatsonis CA, Glasziou PP, Irwig LM, Lijmer JG, Moher D, Rennie D, de Vet HC: Towards complete and accurate reporting of studies of diagnostic accuracy: the STARD initiative. BMJ 2003, 326:4I-4.

\section{Pre-publication history}

The pre-publication history for this paper can be accessed here: http://www.biomedcentral.com/1471-2474/8/102/pre pub
Publish with Bio Med Central and every scientist can read your work free of charge

"BioMed Central will be the most significant development for disseminating the results of biomedical research in our lifetime. "

Sir Paul Nurse, Cancer Research UK

Your research papers will be:

- available free of charge to the entire biomedical community

- peer reviewed and published immediately upon acceptance

- cited in PubMed and archived on PubMed Central

- yours - you keep the copyright

Submit your manuscript here:

http://www.biomedcentral.com/info/publishing_adv.asp
BioMedcentral 